\title{
What mimics the reflection effect in symbiotic binaries?^
}

\author{
A. Skopal \\ Astronomical Institute, Slovak Academy of Sciences, 05960 Tatranská Lomnica, Slovakia \\ e-mail: skopal@ta3.sk \\ Received 17 February 2000 / Accepted 19 October 2000

\begin{abstract}
We discuss the origin of a periodic wave-like variation as a function of the orbital phase observed in the light curves (LC) of symbiotic stars. It is shown that this type of variability cannot be ascribed to a reflection effect. For example, the observed amplitudes of the LCs are far larger than those calculated within a model of the reflection effect. Here, the nature of the orbitally related changes in the optical continuum is outlined within an ionization model of symbiotic binaries.
\end{abstract}

Key words. stars: binaries: symbiotic

\section{Introduction}

Symbiotic stars are interacting binaries consisting of a cool giant and a hot compact star. Typical orbital periods run between 1-3 years. The giant component losses mass, part of which is accreted by its companion. The hot star ionizes a portion of the giant's wind, giving rise to nebular emission. As a result the spectrum of symbiotic stars consists of basically three components of radiation - two stellar and one nebular. Such composition of the spectrum was demonstrated well for Z And, BF Cyg and AG Dra (Nussbaumer \& Vogel 1989; Fernandéz-Castro et al. 1990; Greiner et al. 1997). In many cases the nebular radiation dominates the optical.

A very significant feature of LCs of many symbiotic stars is a periodic wave-like variation along the orbital motion (see Fig. 1). It displays a large amplitude (i.e. magnitude difference between the maximum and minimum of the light), $\Delta m \sim 1 \mathrm{mag}$ or more, which is a function of the wavelength - we always observe $\Delta U>\Delta B \gtrsim \Delta V$. The period is approximately equal to the orbital period, and a minimum occurs at/around the inferior conjunction of the cool component. These properties relate this type of variations to the orbital motion. Originally, Boyarchuck (1966) and Belyakina (1970) suggested a reflection effect as responsible for such variability in the AGPeg LC. In this model, the hot star irradiates and heats up the facing giant's hemisphere that causes variation in the star's brightness when viewing the binary at different orbital phases. This natural explanation was adopted by many

\footnotetext{
* Appendices A, B, C are only available in electronic form at http://www.edpsciences.org
}

authors (e.g. Kenyon 1986) and it is still popular (e.g. Munari 1989, 1992; Dobrzycka et al. 1993; Proga et al. 1996, 1998). On the other hand, some authors have never used the reflection effect and/or found it to be problematic to interpret their data. For example, Nussbaumer \& Vogel (1987) suggested a new approach to symbiotic stars based on the interaction of the hot star radiation with the cool stellar wind. Formiggini \& Leibowitz (1990) found that the reflection effect requires an extremely high bolometric luminosity of the hot component, to explain the light variation in AG Dra, AX Per and AG Peg. Schmutz et al. (1994) did not find any irradiation effect in the red giant spectrum of SYMus, although its visual LC varies with an amplitude of 0.6 mag. Recently Skopal (1996) demonstrated that the reflection effect is not a reliable interpretation of the wave-like variation in the optical continuum of V443 Her.

Accordingly we show that the reflection effect cannot explain the orbitally related wave-like modulation of LCs of symbiotic binaries. To demonstrate this, we compare basic observational properties of this variation to those caused by the reflection effect (Sect. 3). In Sect. 4 we describe a simple ionization model, which could be responsible for such variation.

\section{Observation}

Figure 1 shows examples of the periodic wave-like variation in the $U B V$ LCs for selected symbiotic stars. Table 1 summarizes its basic characteristics - the amplitude, $\Delta m$, and the profile through a parameter a (Sect. 3.2). Photometric $U B V$ observations used in this paper has been extracted from the literature and are also referred in Table 1. 

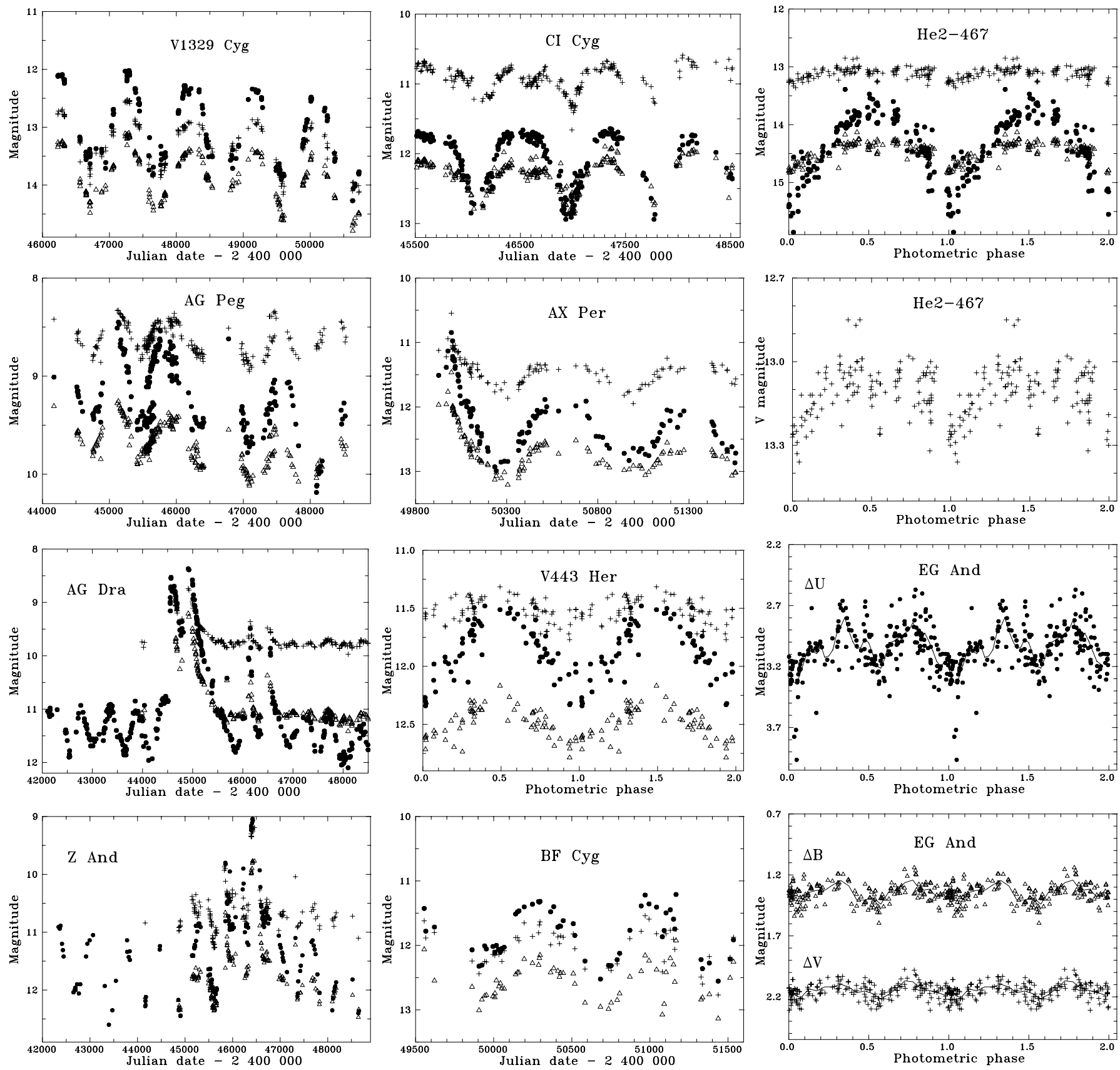

Fig. 1. Examples of periodic wave-like variation in the LCs of selected symbiotic binaries. Symbols, $\bullet, \triangle$ and + , represent observations in the $U, B$ and $V$ band, respectively. Profiles of the LCs are characterized by the parameter $a \sim 0.5$ (left panels), $0.5<a<1$ (mid) and $a \approx 1$ (right), see Sect. 3.2. In cases of a poor coverage of orbital cycles, the data were folded according to their photometric ephemerides. Sources of the data are given in Table 1

\section{Properties of the wave-like variation}

In this section we compare the observed parameters of the wave-like variation - its amplitude and profile - to those given by the reflection effect.

\subsection{The amplitude of light curves}

The largest amplitude, between $\sim 0.5$ and $\sim 2.5 \mathrm{mag}$, is observed in the $U$ band (Fig. 1, Table 1). In the model of the reflection effect, it is given by the luminosity ratio of the illuminated to the non-illuminated giant's hemisphere. The upper limit $\left(i=90^{\circ}\right)$ of the magnitude difference between the two hemispheres, $\Delta m_{\max }$, can be expressed as

$\Delta m_{\max }=-2.5 \log \left(1+2 L_{\mathrm{RE}} / L_{\mathrm{g}}\right)$,

where $L_{\mathrm{g}}$ is the luminosity of the giant and $L_{\mathrm{RE}}$ represents the portion of the hot component luminosity, $L_{\mathrm{h}}$, impacting the giant. If the separation between the stars, $A$, is significantly larger than the giant's radius, $R_{\mathrm{g}}$, then $L_{\mathrm{RE}}=\left(R_{\mathrm{g}} / 2 A\right)^{2} \times L_{\mathrm{h}}$, and Eq. (1) reads as

$\Delta m_{\max }=-2.5 \log (1+\beta / 2)$, 
Table 1. Characteristics of the LC profile: the observed amplitude, $\Delta m$, and the shape, $a$, (Sect. 3.2)

\begin{tabular}{lcccccccccc}
\hline Object & $P_{\text {orb }}$ & $\beta^{\star}$ & $\Delta U$ & $\Delta B$ & $\Delta V$ & $a_{U}$ & $a_{B}$ & $a_{V}$ & Obs. & Note \\
\hline AX Per & 680 & $0.02 \pm 0.008$ & 0.8 & 0.6 & 0.5 & 0.6 & 0.7 & 0.8 & 1 & \\
BF Cyg & 757 & $0.4 \pm 0.12$ & 1.1 & 0.9 & 0.8 & 0.5 & 0.6 & 0.6 & 2 & $s s$ \\
CI Cyg & 855 & $0.06 \pm 0.011$ & 1.2 & 0.7 & 0.5 & 0.8 & 0.8 & 0.9 & 3 & $s s$ \\
EG And & 482 & $\leq 0.002 \pm 0.001$ & 0.6 & 0.3 & 0.2 & 1.0 & 1.0 & 1.0 & 4 & $s s s$ \\
AG Dra & 550 & $0.01 \pm 0.008$ & 1.0 & 0.2 & 0.1 & 0.5 & 0.5 & 0.5 & 5 & \\
V1329 Cyg & 958 & $0.18-0.47$ & 1.8 & 1.3 & 1.2 & 0.5 & 0.5 & 0.5 & 6 & \\
He2-467 & 479 & $0.07-0.2$ & 2.2 & 0.6 & 0.3 & 0.6 & 0.6 & 1.0 & 7 & $s$ \\
Z And & 759 & $\lesssim 0.06 \pm 0.02$ & 1.5 & 1.0 & 0.7 & 0.5 & 0.5 & 0.6 & 8 & $s s$ \\
V443 Her & 594 & $\sim 0.04$ & 0.7 & 0.4 & 0.3 & 0.6 & 0.7 & 0.8 & 9 & \\
AG Peg & 813 & $\sim 0.03$ & 1.1 & 0.6 & 0.5 & 0.5 & 0.5 & 0.5 & 10 & \\
\hline
\end{tabular}

$\star$ - see Appendix A for more detail.

Note: $s$ - secondary minimum in $V$, ss - occasional presence of secondary minima in $V$, sss - secondary minima in $U, B, V$.

Obs.: 1 - Skopal (1998b), Skopal et al. (2000), 2 - Hric et al. (1994, 1996), Skopal et al. (1995, 1997), Skopal (1998b), 3 - Belyakina $(1979,1984,1992), 4$ - Skopal (1997), 5 - Hric et al. (1991, 1993, 1994), Kaler (1987), Kaler et al. (1987), Skopal et al. (1992, 1995), 6 - Arkhipova \& Ikonnikova (1989), Chochol et al. (1999), 7 - Arkhipova \& Noskova (1985, 1987, 1988), 8 - Belyakina (1986, 1992), 9 - Taranova \& Yudin (1987), Belyakina (1992), Kolotilov et al. (1995), 10 - Belyakina (1965, 1970, 1992), Komárek (1990), Fernie (1985), Luthardt (1989), Tomov \& Tomova (1998).

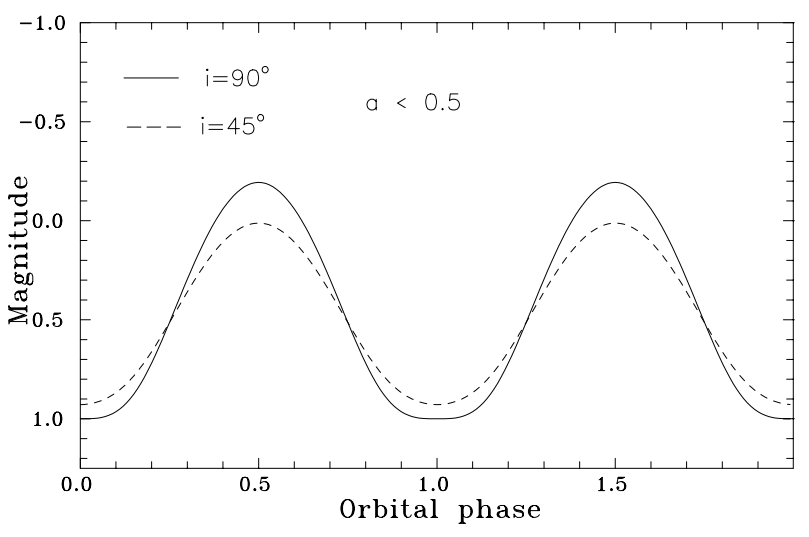

Fig. 2. Profiles of LCs given by the reflection function for inclination of the orbit $i=90^{\circ}$ and $45^{\circ}$ and limb darkening coefficient $u=0.6$. They are characterized by the parameter $a \lesssim 0.5$ (Sect. 3.2 )

where the parameter

$\beta=\frac{R_{\mathrm{g}}^{2}}{A^{2}} \frac{L_{\mathrm{h}}}{L_{\mathrm{g}}}$

measures the strength of the illuminating radiation field relative to that of the giant. So the amplitude of the LC caused by the reflection effect is determined only by the parameter $\beta$. Because of its importance in defining the investigated amplitude we also determined its uncertainties in the cases under discussion (Appendix A). Results are summarized in Table 1 . We can see that the parameter $\beta$ determined from observations can produce a maximum magnitude difference $\Delta m_{\max }<0.1 \mathrm{mag}$, which is far below the observed quantities. On the other hand, the reflection effect (Eq. 2) requires $\beta \geq 1-10$ to match the observed amplitudes.

However, this problem should be treated by solving the radiation transfer of a very hot light $\left(T_{\star} \sim 10^{5} \mathrm{~K}\right)$ throughout a very cool atmosphere $\left(T_{\text {eff }} \sim 3000 \mathrm{~K}\right)$ to learn how the reprocessed $U V / E U V$ radiation contributes into the Balmer and Paschen continua. An exact approach to the reflection effect was outlined by Vaz \& Nordlund (1985) and Nordlund \& Vaz (1990) for similar effective temperatures of the component stars, but not for very cool stars, where the TiO absorption bands are important. Recently, Proga et al. (1996) treated this problem for symbiotic binary stars using a non-LTE photoionization model, but also without including molecules. According to their model, significant changes in the structure of the red giant atmosphere are expected for $\beta \gg 1$ and negligible when $\beta \ll 1$. Their calculations showed that the magnitude difference between the illuminated and nonilluminated hemisphere $\Delta K \lesssim \Delta V \lesssim \Delta B \lesssim \Delta U<$ $0.3 \mathrm{mag}$ in the range of considered temperatures between 20000 and $200000 \mathrm{~K}$ for $\beta=10,1,0.1$ and 0.01 (cf. their Fig. 6). Also according to this study, the theoretical differences in broadband magnitudes between the opposite hemispheres of an illuminated red giant are also very small, $\sim 0.0-0.1 \mathrm{mag}$, far from those observed.

\subsection{The shape of light curves}

To characterize the shape of the observed LC we introduce a parameter $a$ as

$a=\frac{m(0)-m(0.25)}{\Delta m_{\max }}$,

where $m(0)$ and $m(0.25)$ are the magnitudes at the orbital phases 0 and 0.25 , respectively, and $\Delta m_{\max }$ is the amplitude of the LC. The shape of the LC resembles a sinusoidal curve for $a=0.5$, but $a>0.5$ implies a broader maximum than minimum. We can recognize 3 types of LCs, characterized by (i) $a \sim 0.5$ (e.g. V1329 Cyg, AG Peg, left panels of Fig. 1), (ii) $0.5<a<1$ (e.g. CI Cyg, AX Per, mid panels of Fig. 1 ) and (iii) $a \approx 1$ (e.g. He2-467, EG And, right panels of Fig. 1). In some cases the parameter $a$ depends 
on wavelength: $a_{V}>a_{B} \gtrsim a_{U}$ (Table 1 ). Note that extreme values of $a \sim 1$ indicate the possible presence of a secondary minimum in the LC.

Shaping of LCs caused by the reflection effect is determined by a reflection function. To construct such a LC we assume that the observed luminosity of the binary, $L(\epsilon)$, is given by the sum of a constant part of the system luminosity, $L_{\text {const }}$, given mainly by stellar components, and a phase dependent variable light, $L_{\mathrm{v}}(\epsilon)$, given by the reflection function. Then we can write

$L(\epsilon)=L_{\text {const }}+L_{\mathrm{v}}(\epsilon)$,

where $\epsilon=\arccos (\sin i \cos \theta)$ is the angle between the line of sight and the direction to the hot component, the phase angle $\theta$ is determined by the position of the hot star on its relative orbit around the cool giant and $i$ is the orbital inclination. The observed magnitude of the system then can be expressed as

$m(\epsilon)=-2.5 \log \left[1+\frac{L_{\mathrm{v}}(\epsilon)}{L_{\text {const }}}\right]+m_{\text {const }}$.

In our calculations we adopted the reflection function derived by Hadrava (1992). Figure 2 shows example of LCs given by Eq. (6). We can see that the reflection effect produces a strictly periodic modulation of the light along the orbital cycle characterized by the parameter $a \lesssim 0.5$. Therefore the observed profiles characterized with $0.5<a \lesssim 1$ cannot be reproduced by the reflection effect. In addition, recently revealed systematic variation in the minima positions in the LCs of symbiotic binaries (Skopal 1998a) also argues against the location of the main source of the optical continuum on the giant's hemisphere.

We conclude that the observational characteristics of the LCs of symbiotic binaries - the large amplitude, the profile of minima and variation in their positions - cannot be reproduced by the reflection effect.

\section{What mimics the reflection effect in symbiotic binaries?}

To get a better idea of the origin of the orbitally related wave-like variation we discuss it within the basic model of symbiotic binaries - the cool giant losing material via the stellar wind and the hot luminous compact object ionizing a portion of the neutral wind. Such composition creates a strong source of nebular emission in the binary, which often dominates the optical region (e.g. BF Cyg, AG Dra, He2-467, AS 338). Therefore we discuss the apparent variation in the optical continuum within an ionization model.

First, we introduce the simplest model of the ionization structure in symbiotic binaries.

\subsection{A zero level ionization model}

The extent of the ionized zone can be obtained from a parametric equation

$f(r, \vartheta)-X=0$,

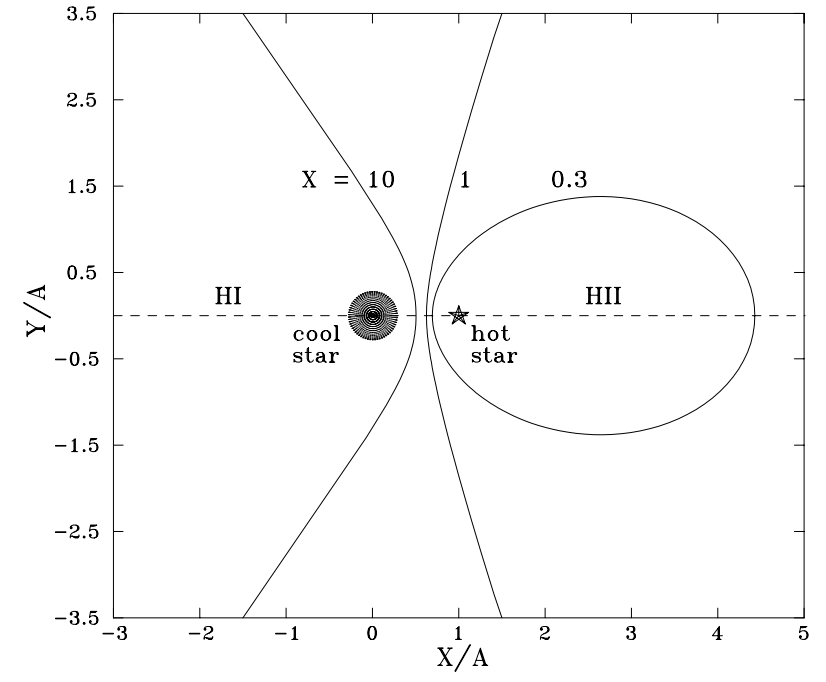

Fig. 3. The $\mathrm{H}_{\mathrm{I}} / \mathrm{H}_{\mathrm{Ii}}$ boundary calculated for $X=0.3,1,10$, the stellar wind model characterized by the parameters $\gamma=$ 2.5 and $R_{\mathrm{g}} / A=0.28$

the solution of which defines the boundary between neutral and ionized gas at the orbital plane determined by a system of polar coordinates, $r, \vartheta$, with the origin at the hot star. The function $f(r, \vartheta)$ was treated for the first time by Seaquist et al. (1984), hereafter STB, for a steady state situation and pure hydrogen gas. The parameter $X$ is given mainly by the binary properties - separation of the components, number of hydrogen ionizing photons, terminal velocity of the wind, $v_{\infty}$, and the mass-loss rate (for details see STB, Nussbaumer \& Vogel 1987). The particle density of the ionized material is given by the velocity distribution of the giant's wind, which is assumed to be of the form

$v_{\text {wind }}=v_{\infty}(1-R / r)^{\gamma}$,

where $r$ is the distance from the centre of the cool star, and $R$ is the origin of the stellar wind ( $\approx$ the radius of the giant). Figure 3 shows examples of the $\mathrm{HI} / \mathrm{H}$ in boundary for $X=0.3,1,10$ and the parameter $\gamma=2.5$ in the wind model (8). STB used $\gamma=0$, which represents a constant velocity of the wind $\left(=v_{\infty}\right)$; no acceleration zone above the giant's photosphere exists. However, stellar wind characterized by the parameter $\gamma>0$ will accelerate gradually above the photosphere to its terminal velocity; it is a more realistic model. Therefore, according to Schröder (1985), we adopted $\gamma=2.5$ in our calculations. In this model, the source of the nebular radiation is physically displaced from the cool giant photosphere.

We now test if the amount of emission produced by this model is consistent with observations.

\subsection{Emission measure}

We investigate the balance between the observed nebular emission in the continuum and that produced by the ionization model. The nebular flux largely depends on the number of hydrogen recombinations, and is proportional 
Table 2. Observed and calculated EM for selected objects

\begin{tabular}{|c|c|c|c|c|c|c|}
\hline Object & $\begin{array}{c}F_{\lambda}^{\mathrm{obs}} / 10^{-13} \\
{\left[\mathrm{erg} \mathrm{cm}^{-2} \mathrm{~s}^{-1} \AA^{-1}\right]}\end{array}$ & Ref. & $\begin{array}{c}\int_{V} n^{2} \mathrm{~d} V \text { (Eq. 9) } \\
{\left[\mathrm{cm}^{-3}\right]}\end{array}$ & $\begin{array}{c}L_{\mathrm{ph}} / \alpha_{B}(\mathrm{Eq} \cdot 10) \\
{\left[\mathrm{cm}^{-3}\right]}\end{array}$ & $\begin{array}{c}\int_{V} n^{2} \mathrm{~d} V \text { (Eq. 13) } \\
{\left[\mathrm{cm}^{-3}\right]}\end{array}$ & $\begin{array}{c}\int_{V} n^{2} \mathrm{~d} V^{c} \\
{\left[\mathrm{~cm}^{-3}\right]}\end{array}$ \\
\hline Z And & $7.5^{a}$ & 1 & $3.410^{59}$ & $2.910^{59}$ & $1.710^{59}$ & \\
\hline Z And & $2.4-9.6^{b}$ & Fig. 1 & $1.9-7.510^{59}$ & & & $1.9-6.010^{59}$ \\
\hline BF Cyg & $5.0^{a}$ & 2 & $3.810^{60}$ & $2.910^{60}$ & $d$ & \\
\hline BF Cyg & $2.7-7.9^{b}$ & Fig. 1 & $3.6-1010^{60}$ & & & $2.6-5.310^{60}$ \\
\hline AG Dra & $2.4^{a}$ & 3 & $1.510^{59}$ & $1.510^{59}$ & $1.110^{59}$ & \\
\hline AG Dra & $0.9-2.2^{b}$ & Fig. 1 & $0.5-1.310^{59}$ & & & $3.5-8.010^{58}$ \\
\hline AX Per & $2.0^{a}$ & 4 & $2.110^{59}$ & $1.210^{59}$ & $d$ & \\
\hline AX Per & $1.0-2.5^{b}$ & Fig. 1 & $1.8-4.610^{59}$ & & & $1.6-5.510^{59}$ \\
\hline V443 Her & $2.1^{a}$ & 5 & $3.010^{59}$ & $4.210^{59}$ & $1.910^{59}$ & \\
\hline V443 Her & $1.1-2.1^{b}$ & Fig. 1 & $2.7-5.210^{59}$ & & & $1.0-2.010^{59}$ \\
\hline
\end{tabular}

$a$ - from the energy distribution in the spectrum at $\lambda 3646^{-} \AA$.

$b$ - from the dereddened $U$-magnitude at minimum and maximum, respectively:

Z And: $U=12.3-10.8, E_{B-V}=0.35, d=1.12 \mathrm{kpc}$, BF Cyg: $U=12.4-11.3, E_{B-V}=0.4, d=4.6 \mathrm{kpc}$, AG Dra: $U=12.0-11.0$, $E_{B-V}=0.05, d=1.0 \mathrm{kpc}\left(\right.$ Appendix A), AX Per: $U=12.9-11.9, E_{B-V}=0.27, d=1.73 \mathrm{kpc}$, V443 Her: $U=12.2-11.5$, $E_{B-V}=0.15, d=2.0 \mathrm{kpc}$.

$c$ - the range of the observed $E M$ referred in the literature.

$d$ - not applicable.

Ref.: 1 - Fig. 1 of Fernández-Castro et al. (1988), 2 - Fig. 3b of Fernández-Castro et al. (1990), Skopal et al. (1997), 3 - Fig. 3 of Mikolajewska et al. (1995), Appendix A, 4 - Fig. 2 of Mikolajewska \& Kenyon (1992), Skopal (2000), 5 - Fig. 1 of Dobrzycka et al. (1993).

to $\int n_{+} n_{\mathrm{e}} \mathrm{d} V$, (the so called emission measure - EM); $n_{+}$ and $n_{\mathrm{e}}$ is the concentration of ions (protons) and electrons, respectively.

(i) Observations: The quantity of the $E M$ can be estimated, for example, from the measured flux, $F_{\lambda}^{\text {obs }}$ ( $\operatorname{erg~cm}{ }^{-2} \mathrm{~s}^{-1} \AA^{-1}$ ), of the nebular continuum at the wavelength $\lambda$, according to the equation

$4 \pi d^{2} F_{\lambda}^{\mathrm{obs}}=\varepsilon_{\lambda} \int_{V} n_{+} n_{\mathrm{e}} \mathrm{d} V$,

in which $d$ is the distance to the object, $\varepsilon_{\lambda}$ is the volume emission coefficient per electron and per ion

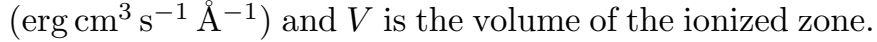
The nebular flux can be obtained from the energy distribution in the spectrum. Its upper limit can also be estimated from the dereddened $U$-magnitude of systems, in which the nebular continuum dominates the optical.

(ii) Model: The source of the nebular radiation in the model is the ionized region, in which the rate of ionization/recombination processes is balanced by the rate of photons, $L_{\mathrm{ph}}$ (photons $\mathrm{s}^{-1}$ ), capable of ionizing the element under consideration. In the case of pure hydrogen we can write the equilibrium condition as

$L_{\mathrm{ph}}=\alpha_{B} \int_{V} n_{+}(r) n_{\mathrm{e}}(r) \mathrm{d} V$,

where $\alpha_{B}\left(\mathrm{~cm}^{3} \mathrm{~s}^{-1}\right)$ is the total hydrogenic recombination coefficient. The number of hydrogen ionizing photons, $L_{\mathrm{ph}}$ is

$L_{\mathrm{ph}}=\frac{L_{\mathrm{h}}}{\sigma T_{\star}^{4}} \pi(h c)^{-1} \int_{0}^{912} \lambda B_{\lambda}\left(T_{\star}\right) \mathrm{d} \lambda$, where $L_{\mathrm{h}}\left[\operatorname{erg~s}^{-1}\right]$ and $T_{\star}$ is the total luminosity and the temperature of the hot star, respectively. Having independently determined $L_{\mathrm{ph}}$ we compare the $E M$ given by observations (Eq. 9) and that required by the ionization model (Eq. 10). In Table 2 we give results for the objects with available parameters. We obtained the observed fluxes from the energy distribution in the spectrum at $\lambda 3646^{-} \AA$ and used the hydrogen emission coefficient $\varepsilon_{3646^{-}}=3.3610^{-28} \mathrm{erg} \mathrm{cm}^{3} \mathrm{~s}^{-1} \AA^{-1}$ (Gurzadyan 1997). For a comparison, we also estimated the nebular flux from the $U$-magnitude according to the calibration of Henden \& Kaitchuck (1982). In this case we adopted $\varepsilon_{U}=1.910^{-28} \mathrm{erg} \mathrm{cm}^{3} \mathrm{~s}^{-1} \AA^{-1}$ as the average of $\varepsilon_{3646^{-}}$ and $\varepsilon_{3646+}$.

We can calculate the $E M$ directly by integrating emission contributions throughout the volume of the ionized zone defined by the model (Eq. 7). The calculation of the $\mathrm{H} \mathrm{I} / \mathrm{H}$ II and He I/He II boundary in 2-D representation can be found in STB and Nussbaumer \& Vogel (1987), respectively. Using Nussbaumer \& Vogel (1989), we derive an upper limit to the modeled $E M$ assuming the sphere around the cool star to be fully ionized from $r=Q$ to $r=\infty$. The parameter $Q$ is the location of the $\mathrm{HI} / \mathrm{H}$ II boundary on the line joining the cool and the hot star $\left(Q>R \sim R_{\mathrm{g}}\right)$. The particle density $n(r)$ is given by the mass-loss rate of the wind

$\dot{M}=4 \pi r^{2} \mu m_{\mathrm{H}} n(r) v_{\text {wind }}$,

where $\mu$ is the mean molecular weight, $m_{\mathrm{H}}$ is the mass of the hydrogen atom and the velocity of the wind is given by 
Eq. (8). Then the EM for a completely ionized medium $\left(n_{\mathrm{e}}=n_{+}\right)$can be calculated from the following analytical expression

$$
\begin{aligned}
& \int_{V} n(r)^{2} \mathrm{~d} V=4 \pi\left[\frac{\dot{M}}{4 \pi \mu \mathrm{m}_{\mathrm{H}} v_{\infty}}\right]^{2} \int_{Q}^{\infty} \frac{\mathrm{d} r}{r^{2}(1-R / r)^{2 \gamma}} \\
& =4 \pi\left[\frac{\dot{M}}{4 \pi \mu \mathrm{m}_{\mathrm{H}} v_{\infty}}\right]^{2} \frac{1}{R(1-2 \gamma)}\left[1-\left(1-\frac{R}{Q}\right)^{1-2 \gamma}\right] \\
& \text { for } \gamma \neq 0.5 \\
& \text { and }
\end{aligned}
$$

$$
=-4 \pi\left[\frac{\dot{M}}{4 \pi \mu \mathrm{m}_{\mathrm{H}} v_{\infty}}\right]^{2} \frac{1}{R} \ln \left(1-\frac{R}{Q}\right) \quad \text { for } \gamma=0.5 \text {. }
$$

Nussbaumer \& Vogel (1989) derived formula (13) for the special case of $\gamma=2.5$. This approximation can be used for cases when the major part of the circumbinary environment is ionized, i.e. the parameter $X>10$ (Fig. 3). We applied the formula (13) to Z And, AG Dra and V443 Her (Col. 6 in Table 2). The calculated $E M$ is somewhat lower than that indicated by observations. However, given the large uncertainties in fundamental parameters (mainly in $d, \dot{M}$ and $L_{\mathrm{h}}$, see Appendix A) we still consider the calculated values to be consistent with observations. However, if the calculated EM according to Eq. (13) is really lower than that measured, this could imply the presence of an additional source of particles in the system - for example, the hot star wind. Note that in the case of an open ionized zone a portion of the $L_{\mathrm{ph}}$ photons escapes the system, and thus an injection of new particles (emitters) into such a zone will produce an extra flux.

We find that the observed EM is consistent with that produced by the ionization model. This implies that all the $L_{\mathrm{ph}}$ photons consumed by the particles of the giant wind are needed to produce the observed nebular flux. However, in the model of the reflection effect only a small part of ionizing photons, $\left(R_{\mathrm{g}} / 2 A\right)^{2} \times L_{\mathrm{ph}}$ (Sect. 3), can be used to produce the nebular radiation. This causes the discrepancy between the $\beta$ parameter given by observations and much larger value, required by models of reflection effect to explain the amplitude of the LCs.

We now will demonstrate that the variation in the $E M$ is responsible for the investigated wave-like variation in the LCs.

\subsection{Variation in the emission measure}

The quantity of the $E M$ in the continuum also varies as a function of the orbital phase (e.g. Fernandez-Castro et al. 1988; Mikolajewska et al. 1989; Mikolajewska \& Kenyon 1992; Dobrzycka et al. 1993). To investigate this variability we express Eq. (9) in the scale of magnitudes $\left(m_{\lambda}=-2.5 \log \left(F_{\lambda}\right)+q_{\lambda}\right)$ as

$m_{\lambda}=-2.5 \log (E M)+C_{\lambda}$,

where

$C_{\lambda}=q_{\lambda}-2.5 \log \left(\frac{\varepsilon_{\lambda}}{4 \pi d^{2}}\right)$, in which the constant $q_{\lambda}$ defines magnitude zero. For the standard photometry and fluxes in units of $\operatorname{erg} \mathrm{cm}^{-2} \mathrm{~s}^{-1} \AA^{-1}, q_{U}=-20.9, q_{B}=-20.36$ and $q_{V}=$ -21.02 (Henden \& Kaitchuck 1982). Using Eq. (14) we constructed the LCs from the measured values of the $E M$ in BF Cyg and $\mathrm{Z}$ And and compared them to those obtained photometrically. We calculated the $B$ magnitudes, because in these objects the nebular contribution still dominates the continuum and the emission coefficient is nearly constant in this region. We used $\varepsilon_{B}=0.510^{-28} \mathrm{erg} \mathrm{cm}^{3} \mathrm{~s}^{-1} \AA^{-1}$ (Gurzadyan 1997) and $d=4.6$ and $1.12 \mathrm{kpc}$ for BF Cyg and Z And, respectively. Figure 4 shows that the variation in the $E M$ follows well that observed in the LCs. In other systems, where only a few measurements of the $E M$ at different orbital phases are available (AX Per, AG Dra, V443 Her), we estimated the amplitude as

$\Delta m_{E M}=-2.5 \log \frac{E M_{\min }}{E M_{\max }}$.

We find that the observed range of the $E M$ is comparable with the amplitude of LCs in the $U$ band of these systems (the last column in Table 2). We conclude that the variation in the EM produces the observed phase-dependent variation in the LCs of symbiotic binaries.

Note that the decrease of the LC amplitude with wavelength is caused by an increase of the cool giant contribution, which does not vary with the orbital phase. The same effect takes place towards the short wavelengths due to an increase in the stellar contribution from the hot star (see Fig. 4 of Kenyon et al. 1993).

\subsection{Variation in the hydrogen lines}

Together with the periodic variation in the nebular continuum, the same type of variability is observed in fluxes of Balmer lines. The source of hydrogen emission in lines is also the $\mathrm{H}$ II region. Therefore the variation in both the hydrogen continuum and the lines should be of the same nature. So the $E M$ derived from the hydrogen continuum should be consistent with that given by the Balmer lines, assuming an optically thin regime and the case $B$ of recombination. In Table 3 we summarize results for the line $\mathrm{H} \beta$. We calculated the $E M_{\beta}$ according to Eq. (9) using the volume emission coefficient in the $\mathrm{H} \beta$ line, $\varepsilon_{\beta}=1.2310^{-25} \mathrm{erg} \mathrm{cm}^{3} \mathrm{~s}^{-1}$. We can see that the $E M_{\beta}$ is by a factor of $\sim 5$, on average, lower than that derived from the Balmer continum. This discrepancy can be caused by atenuation of the $\mathrm{H}$ I emission due to (i) an absorption originating from the cool component wind and (ii) the self-absorption effect, when the nebula is partially opaque in the line $\mathrm{H} \beta$ and $\mathrm{H} \gamma$, in addition to the case $B$. The first case is demonstrated well by sets of observations along the orbital cycle (e.g. Oliversen et al. 1985). The wind absorption component is more pronounced around the inferior conjunction of the giant (see Fig. 3), which makes the amplitude of the variation in line fluxes even larger. The second case is indicated by the observed very 

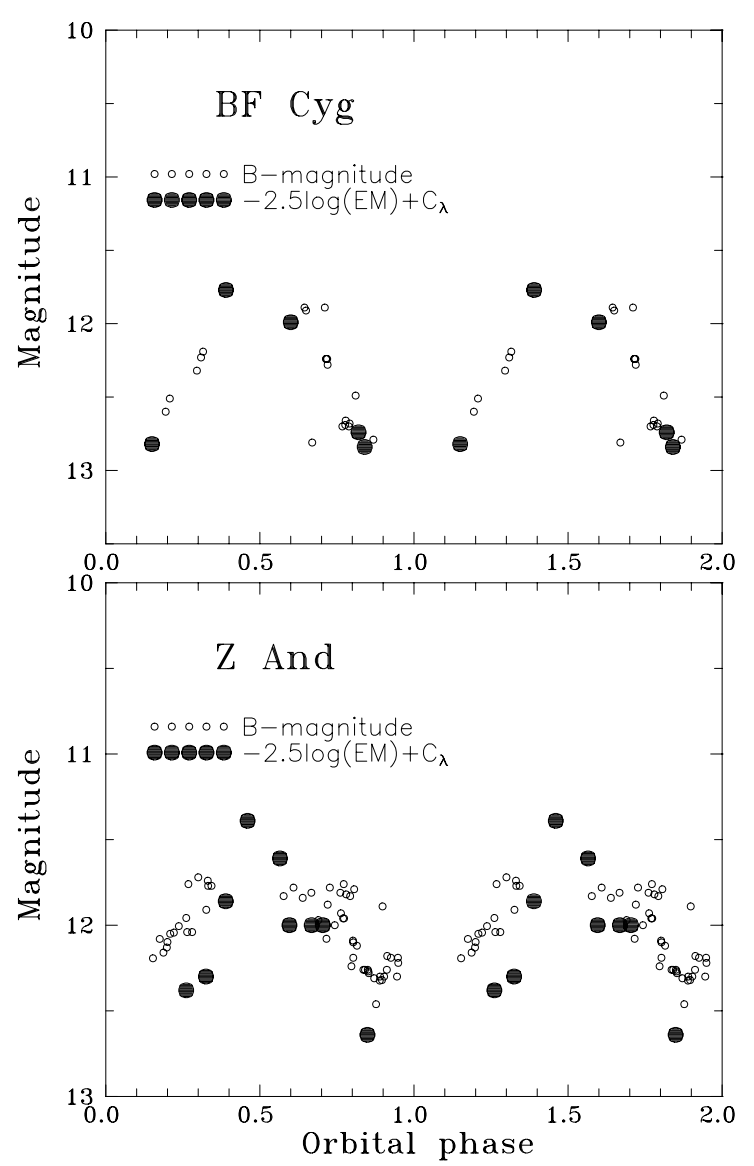

Fig. 4. Top: variation in the $E M$ of $\mathrm{BF}$ Cyg as a function of the orbital phase. The data (Mikolajewska et al. 1989) were converted into the $B$-magnitudes according to Eq. (14). Compared is the LC in the $B$ band obtained photometrically during the same period, between JD 2445700 and 2446718 (Hric et al. 1993). Bottom: the same as the top, but for Z And. Measurements of the $E M$ were taken from Fernandez-Castro et al. (1988), and photometric $B$-magnitudes from Fig. 1, but omitting the active phase. These results show that the variation in the $E M$ is fully responsible for the variation in the LCs

steep Balmer decrement $(\mathrm{H} \alpha / \mathrm{H} \beta / \mathrm{H} \gamma \approx 7 / 1 / 0.4$, for objects in Table 3 ), which is not consistent with the theoretical prediction $(2.85 / 1 / 0.47)$ that the excitation is due to photoionization. Moreover, attenuation of hydrogen fluxes could also be in part due to electron collisions in a dense $\mathrm{H}$ II region, and/or partly caused by selective interstellar absorption. Therefore the $E M_{\beta}$ represents only the lower limit of the $E M$ derived from the Balmer continuum.

As the variation in the emission of the Balmer lines is connected with the $\mathrm{H}$ II region - the dominant source of the optical/near-UV continuum - the observed periodic wave-like variation in the continuum should always be followed by a similar variation in Balmer lines.

\subsection{Why does the emission measure vary?}

To produce the wave-like variation in the LCs along the orbital cycle, the nebula - the main source of the optical
Table 3. Emission measure in the line $\mathrm{H} \beta$ for selected objects

\begin{tabular}{cccc}
\hline Object & $\begin{array}{c}F_{\beta} / 10^{-12 a} \\
{\left[\mathrm{erg} \mathrm{cm}^{-2} \mathrm{~s}^{-1}\right]}\end{array}$ & Ref. & $\begin{array}{c}E M_{\beta} \\
{\left[\mathrm{cm}^{-3}\right]}\end{array}$ \\
\hline Z And & 37.3 & 1 & $4.510^{58}$ \\
BF Cyg & $18-65$ & 2 & $0.4-1.310^{60}$ \\
AG Dra & $6.4-9.4$ & 3 & $6.2-9.110^{57}$ \\
AX Per & $7.2-22$ & 4 & $2.1-6.310^{58}$ \\
V443 Her & $6.5-15$ & 5 & $2.5-5.710^{58}$ \\
\hline
\end{tabular}

${ }^{a}$ - dereddened fluxes with $E_{B-V}$ referred in Table 2.

Ref.: 1 - Mikolajewska \& Kenyon (1996), 2 - Mikolajewska et al. (1989), 3 - Mikolajewska et al. (1995), 4 - Mikolajewska \& Kenyon (1992), 5 - Dobrzycka et al. (1993).

continuum in symbiotic binaries - has to be partially optically thick and of a non-spherical shape. In our simple ionization model the opacity, $\kappa$, of the ionized emission medium decreases with the distance from the cool star, since $\kappa \propto n \propto r^{-2}$ (i.e. its parts nearest to the giant's surface will be most opaque). It is probable that the observed emission will also depend on the extension of the ionized region. Below we give a qualitative description on how, or whether, it is possible to produce the observed profile of LCs within the ionization model mentioned above.

(i) In the case of an oval shape of the H II zone (Fig. 3; a small parameter $X$ ), its total emission will be atenuated more at positions of the inferior and superior conjunction of the cool star (the orbital phase $\varphi=0$ and 0.5 , respectively) than at positions of $\varphi=0.25$ and 0.75 , respectively. Such apparent variation in the $E M$ will produce both the primary and the secondary minimum in the LC and will thus mimic the ellipsoidal effect in binaries containing a giant star. This type of LC profile corresponds to the parameter $a \sim 1$ (right panels of Fig. 1).

(ii) A gradual opening of the $\mathrm{H}$ II zone (approximately $0.3<X \lesssim 1$ ) will make it optically thinner behind the hot star (outside the binary around $\varphi=0.5$ ). Thus, more of the nebular radiation, relative to the case (i), will be observed at the position of $\varphi \sim 0.5$. The secondary minimum in LCs will therefore become less pronounced or flat, and/or a maximum at $\varphi \sim 0.5$ can arise. The LC profile here should be characterized by the parameter $a>0.5$ (mid panels of Fig. 1).

(iii) Given an extensive emission zone $(X>10)$ one can imagine the partially optically thick portion of the $\mathrm{HII}$ region as a cap on the $\mathrm{HI} / \mathrm{H}$ II boundary around the binary axis. This resembles the geometry of the reflection effect, but the emission region causing the light variation is physically displaced from the giant's surface (see also a sketch in Baratta \& Viotti (1990), who drawn such regions for Fe II and C III lines). In these cases the LC profile is similar to that of the reflection effect, i.e. the parameter $a \leq 0.5$ (left panels of Fig. 1).

A relationship between the parameters $a$ and $X$ supports the connection between the shape of the LC and the 
extent of the symbiotic nebula mentioned above (Fig. B.1, Appendix B).

However, the real structure of the ionized region in symbiotic binaries is probably much more complex. Currently it is being intensively investigated (e.g. Schmid 1998). For example, the observed systematic variation in the minima position (i.e. an apparent change of the orbital period) requires an asymmetrical shape of the $\mathrm{HII}$ zone with respect to the binary axis (see Sect. 4.1 of Skopal 1998a in more detail). A modification of the STB model, which includes effects of the orbital motion, is outlined in Appendix C.

\section{Conclusion}

We find that:

1. The observed properties of the orbitally related variation in the LCs of symbiotic binaries - the large amplitude, shaping of minima and systematic changes in their positions - cannot be produced by the reflection effect.

2. According to the basic ionization model of symbiotic binaries, the main source of the optical continuum is the nebula, which arises from ionization of the cool giant wind by the hot star radiation. We found that:

(a) The observed $E M$ is consistent with that produced by the ionization model.

(b) Variation in the EM is fully responsible for the observed wave-like modulation of the LCs of symbiotic binaries with the orbital phase.

(c) The variation in the optical continuum should always be followed by a similar variation in Balmer lines.

3. To explain the orbitally-related variation, the nebula has to be partially optically thick and of a nonsymmetrical shape to produce different contributions of its total emission into the line of sight at different orbital phases.

4. The LC profile depends on the extent of the symbiotic nebula. Large nebulae $(X \gtrsim 10)$ produce a sinusoidal LC $(a \sim 0.5)$. Less extended nebulae $(X \lesssim 1)$ have a flatter LC at its maximum, and/or create a secondary minimum, $(0.5<a \lesssim 1)$, which mimics the ellipsoidal effect.

Acknowledgements. This research has been supported by the Slovak Academy of Science under a grant No. 5038/2000 and the Alexander von Humboldt foundation under project No. SLA/1039115. The author would like to thank his anonymous referee for constructive comments that allowed him to improve the original version of the present work.

\section{References}

Aller, L. H. 1954, Pub. DAO Victoria, 9, 321

Arkhipova, V. P., \& Ikonnikova, N. P. 1989, Pisma v AZh, 15, 140

Arkhipova, V. P., \& Noskova, R. I. 1985, SvA Lett., 11, 297
Arkhipova, V. P., \& Noskova, R. I. 1987, Astron. Tsirk., 1478 Arkhipova, V. P., \& Noskova, R. I. 1988, SvA Lett., 14, 188

Arkhipova, V. P., Ikonnikova, N. P., \& Noskova, R. I. 1995, Pisma v AZh, 21, 379

Baratta, G. B., \& Viotti, R. 1990, A\&A, 229, 104

Belyakina, T. S. 1965, Izv. Krymsk. Astrofiz. Obs., 33, 226

Belyakina, T. S. 1970, Astrofizika, 6, 49

Belyakina, T. S. 1979, Izv. Krymsk. Astrofiz. Obs., 59, 133

Belyakina, T. S. 1984, Izv. Krymsk. Astrofiz. Obs., 68, 108

Belyakina, T. S. 1986, Izv. Krymsk. Astrofiz. Obs., 75, 136

Belyakina, T. S. 1992, Izv. Krymsk. Astrofiz. Obs., 84, 49

Boyarchuk, A. A. 1966, AZh, 43, 976

Chochol, D., Andronov, I. L., Arkhipova, V. P., et al. 1999, Contr. Astron. Obs. Skalnaté Pleso, 29, 31

Dobrzycka, D., Kenyon, S. J., \& Mikolajewska, J. 1993, AJ, 106,284

Fernández-Castro, T., Cassatella, A., Giménez, A., \& Viotti, R. 1988, ApJ, 324, 1016

Fernández-Castro, T., González-Riestra, R., Cassatella, A., \& Fuensalida, J. J. 1990, A\&A, 227, 422

Fernie, J. D. 1985, PASP, 97, 653

Formiggini, L., \& Leibowitz, E. M. 1990, A\&A, 227, 121

Gális, R., Hric, L., Friedjung, M., \& Petrík, K. 1999, A\&A, 348,533

Greiner, J., Bickert, K., Luthardt, R., et al. 1997, A\&A, 322, 576

Gurzadyan, G. A. 1997, The Physics and Dynamics of Planetary Nebulae (Springer-Verlag, Berlin)

Hadrava, P. 1992, A\&A, 257, 218

Henden, A. A., \& Kaitchuck, R. H. 1982, Astronomical Photometry (Van Nostrand Reinhold Company, New York)

Hric, L., Skopal, A., Urban, Z., et al. 1991, Contr. Astron. Obs. Skalnaté Pleso, 21, 303

Hric, L., Skopal, A., Urban, Z., et al. 1993, Contr. Astron. Obs. Skalnaté Pleso, 23, 73

Hric, L., Skopal, A., Chochol, D., et al. 1994, Contr. Astron. Obs. Skalnaté Pleso, 24, 31

Hric, L., Skopal, A., Urban, Z., et al. 1996, Contr. Astron. Obs. Skalnaté Pleso, 26, 46

Kaler, J. B. 1987, AJ, 94, 437

Kaler, J. B., Stoehr, C. A., Hartkopf, W. I., et al. 1987, AJ, 94, 452

Kamath, U. S., \& Ashok, N. M. 1999, A\&AS, 135, 199

Kenyon, S. J. 1986, The symbiotic stars (Cambridge Univ. Press, Cambridge), 27

Kenyon, S. J., Oliversen, N. A., Mikolajewska, J., et al. 1991, AJ, 101, 637

Kenyon, S. J., Mikolajewska, J., Mikolajewski, M., et al. 1993, AJ, 106, 1573

Kolotilov, E. A., Munari, U., \& Yudin, B. F. 1995, A\&A, 293, 815

Komárek, Z. 1990, Bull. Astron. Czechosl. Inst., 41, 131

Luthardt, R. 1989, Veröff. Sternwarte Sonne., 10, 255

Mikolajewska, J., Kenyon, S. J., \& Mikolajewski, M. 1989, AJ, 98, 1427

Mikolajewska, J., \& Kenyon, S. J. 1992, AJ, 103, 579

Mikolajewska, J., \& Kenyon, S. J. 1996, AJ, 112, 1659

Mikolajewska, J., Kenyon, S. J., Mikolajewski, M., et al. 1995, AJ, 109, 1289

Munari, U. 1989, A\&A, 208, 63

Munari, U. 1992, A\&A, 257, 163

Munari, U., Margoni, R., \& Mammano, A. 1988, A\&A, 202, 83 
Munari, U., \& Buson, L. M. 1992, A\&A, 255, 158

Mürset, U., Nussbaumer, H., Schmid, H. M., \& Vogel, M. 1991, A\&A, 248, 458

Mürset, U., \& Schmid, H. M. 1999, A\&AS, 137, 473

Nordlund, Å., \& Vaz, L. P. R. 1990, A\&A, 228, 231

Nussbaumer, H., \& Vogel, M. 1987, A\&A, 182, 51

Nussbaumer, H., \& Vogel, M. 1989, A\&A, 213, 137

Oliversen, N. A., Anderson, C. M., Stencel, R. E., \& Slovak, H. M. 1985, ApJ, 295, 620

Pereira, C. B. 1996, ApSS, 235, 305

Proga, D., Kenyon, S. J., Raymond, C., \& Mikolajewska, J. 1996, ApJ, 471, 930

Proga, D., Kenyon, S. J., \& Raymond, C. 1998, ApJ, 501, 339

Schild, H., \& Schmid, H. M. 1997, A\&A, 324, 606

Schmid, H. M. 1998, Mod. Astron., 11

Schmid, H .M., \& Schild, H. 1997, A\&A, 327, 219

Schmutz, W., Schild, H., Mürset, U., \& Schmid, H. M. 1994, A\&A, 288, 819

Schröder, K.-P. 1985, A\&A, 147, 103

Seaquist, E. R., Taylor, A. R., \& Button, S. 1984, ApJ, 284, 202 (STB)

Skopal, A. 1994, A\&A, 286, 453

Skopal, A. 1996, Ap\&SS, 238, 285
Skopal, A. 1997, A\&A, 318, 53

Skopal, A. 1998a, A\&A, 338, 599

Skopal, A. 1998b, Contr. Astron. Obs. Skalnaté Pleso, 28, 87

Skopal, A. 2000, Contr. Astron. Obs. Skalnaté Pleso, 30, 21

Skopal, A., Hric, L., Urban, Z., et al. 1992, Contr. Astron. Obs. Skalnaté Pleso, 22, 131

Skopal, A., Hric, L., Chochol, D., et al. 1995, Contr. Astron. Obs. Skalnaté Pleso, 25, 53

Skopal, A., Vittone, A., Errico, L., et al. 1997, MNRAS, 292, 703

Skopal, A., Pribulla, T., Wolf, M., Shugarov, S., \& Jones, A. 2000, Contr. Astron. Obs. Skalnaté Pleso, 30, 28

Skopal, A., Teodorani, M., Errico, L., et al. 2001, A\&A, in press

Smith, V. V., Cunha, K., Jorissen, A., \& Boffin, H. M. J. 1996, A\&A, 315, 179

Taranova, O. G., \& Yudin, B. F. 1987, Astron. Tsirk., 1489, 7 Tomov, N., \& Tomova, M. 1998, IBVS, No. 4574

van Belle, G. T., Lane, B. F., Thompson, R. R., et al. 1999, AJ, 117, 521

Vaz, L. P. R., \& Nordlund, Å. 1985, A\&A, 147, 281

Vogel, M. 1991, A\&A, 249, 173

Vogel, M., Nussbaumer, H., \& Monier, R. 1992, A\&A, 260, 156 